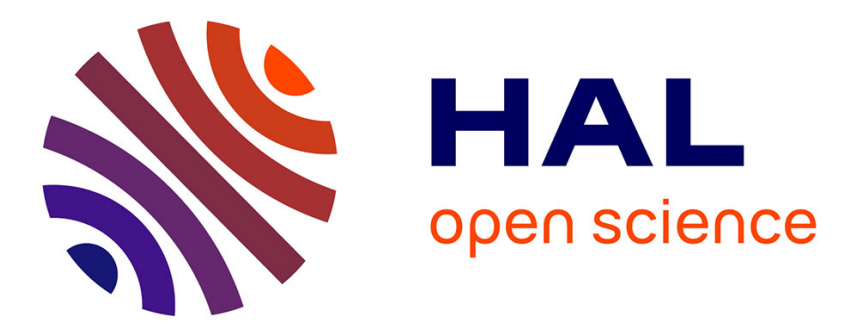

\title{
Parameter estimation from nonlinear frequency response of MEMS resonators
}

\author{
Alexis Brenes, Jérôme Juillard, Laurent Bourgois, Filipe Vinci dos Santos
}

\section{To cite this version:}

Alexis Brenes, Jérôme Juillard, Laurent Bourgois, Filipe Vinci dos Santos. Parameter estimation from nonlinear frequency response of MEMS resonators. 2015 IEEE International Symposium on Circuits and Systems (ISCAS), May 2015, Lisbonne, Portugal. pp.1318-1319, 10.1109/ISCAS.2015.7168884 . hal-01235608

\section{HAL Id: hal-01235608 \\ https://hal-centralesupelec.archives-ouvertes.fr/hal-01235608}

Submitted on 4 Dec 2015

HAL is a multi-disciplinary open access archive for the deposit and dissemination of scientific research documents, whether they are published or not. The documents may come from teaching and research institutions in France or abroad, or from public or private research centers.
L'archive ouverte pluridisciplinaire HAL, est destinée au dépôt et à la diffusion de documents scientifiques de niveau recherche, publiés ou non, émanant des établissements d'enseignement et de recherche français ou étrangers, des laboratoires publics ou privés. 


\section{Parameter estimation from nonlinear frequency response of MEMS resonators}

\author{
A. Brenes \\ THALES Avionics \\ Valence, France \\ alexis.brenes@supelec.fr
}

\author{
J. Juillard ${ }^{1}$, L. Bourgois ${ }^{1}$ and F. Vinci dos Santos ${ }^{2}$ \\ E3S ${ }^{1}$ and THALES Chair ${ }^{2}$ \\ Ecole Supérieure d'Electricité (Supélec) \\ Gif-sur-Yvette, France
}

\begin{abstract}
This paper deals with parameter extraction from nonlinear frequency responses of resonant systems. We show that nonlinear frequency responses can be exploited to identify not only the Q-factor and the natural frequency of a resonator, but also information about its internal structure. The proposed method requires less demanding measurements and is less modeldependent than the existing state-of-the-art methods. Its accuracy is illustrated with simulations and it is experimentally validated on a capacitive MEMS resonator.
\end{abstract}

Keywords-open-loop actuation; nonlinear regime; resonator characterization; MEMS; pulsed-mode actuation.

\section{INTRODUCTION}

In this paper, we analyze and give experimental evidence of accurate parameter extraction from a nonlinear resonant frequency response. We apply our method to the characterization of capacitive MEMS and validate it through experimental results on a one-port high-Q capacitive MEMS.

Commonly, the characterization of a resonator consists in estimating its natural frequency and Q-factor, either via openloop frequency response curves [1], or exponential decay time measurements [2]. These measurements require low-amplitude oscillation regimes and, hence, are hampered by low SNR. Moreover, effects such as creep, fatigue, or dielectric charging are known to impair the performance of resonators during their lifetime [3-5] and can hardly be detected by the single estimation of the Q-factor and the resonance frequency.

In nonlinear regimes, open-loop frequency responses are distorted so that the symmetry of amplitude resonance curves around the natural frequency is broken, sometimes resulting in the emergence of jump discontinuities and bifurcation points. A recent study has demonstrated Q-factor estimation from a nonlinear frequency response [6], via precise measurements at low amplitudes (hence at low SNR) and the knowledge of the maximal amplitude on the frequency response, both of them difficult to measure in practice. Another study has demonstrated the efficiency of nonlinear least-squares fitting for parameter estimation of piezoelectric MEMS resonators from a nonlinear frequency response with jump discontinuities obtained through optical measurements [7]. This last study demonstrated that nonlinear frequency responses reveal more information about a resonator than linear lorentzian responses. However, the method developed in [7] requires a precise measurement of the upper bifurcation point and numerous measurements around the maximal amplitude. These shortcomings gave impulse to our development of a new approach, easier to apply to mass produced resonators.

This paper is organized as follows: In section II, the mathematical background required to perform parameter estimation of a nonlinear response is presented. In Section III, we illustrate parameter estimation on different pseudoexperimental (i.e. simulated) data sets. In Section IV, we adapt our method to a capacitive clamped-clamped MEMS resonator and then validate it experimentally in Section V.

\section{MATHEMATICAL BACKGROUND}

\section{A. Resonator analytical model}

Resonators and MEMS devices exhibit a large variety of nonlinearities, from hardening to softening effects [8]. For example, the behavior of a resonator under sine-wave actuation may be described by:

$$
\frac{d^{2} x}{d t^{2}}+\frac{\omega_{0}}{Q} \frac{d x}{d t}+\omega_{0}^{2} x=G^{\theta}(x)+F^{\theta}(x) \sin (\omega t+\varphi)
$$

where $x$ is the normalized displacement of the resonator, $\omega_{0}$ its natural frequency, $Q$ its $\mathrm{Q}$-factor and $F^{\theta}$ and $G^{\theta}$ normalized forces depending on a vector $\boldsymbol{\theta}$ of parameters. Assuming that $F^{\theta}$ and $G^{\theta}$ only exhibit static memory-less nonlinearities and $x(t)=A \sin \omega t(0<A<1), F^{\theta}(x(t))$ and $G^{\theta}(x(t))$ may be expressed:

$$
\begin{aligned}
& F^{\theta}(x(t))=\sum_{k=0}^{\infty} F_{2 k+1}^{\theta}(A) \sin ((2 k+1) \omega t)+F_{2 k}^{\theta}(A) \cos (2 k \omega t) \\
& G^{\theta}(x(t))=\sum_{k=0}^{\infty} G_{2 k+1}^{\theta}(A) \sin ((2 k+1) \omega t)+G_{2 k}^{\theta}(A) \cos (2 k \omega t)
\end{aligned}
$$

Equation (1) may be analyzed by using the method of harmonic balance [9]. Considering (2), this method yields:

$$
\begin{aligned}
& \left(\omega_{0}^{2}-\omega^{2}\right) A-G_{1}^{\theta}(A)=\tau_{-}^{\theta}(A) \cos \varphi \\
& \frac{\omega \omega_{0} A}{Q}=\tau_{+}^{\theta}(A) \sin \varphi
\end{aligned}
$$


where $\tau_{ \pm}^{\theta}(A)=F_{0}^{\theta}(A) \pm \frac{F_{2}^{\theta}(A)}{2}$

Finally:

$$
\begin{aligned}
& h\left(A, \omega, \omega_{0}, Q, \theta\right)=1- \\
& A^{2}\left[\frac{\left[\left(\omega_{0}^{2}-\omega^{2}\right)-\frac{G_{1}^{\theta}(A)}{A}\right]^{2}}{\tau_{-}^{\theta}(A)^{2}}+\frac{\omega^{2} \omega_{0}^{2}}{Q^{2} \tau_{+}^{\theta}(A)^{2}}\right]=0
\end{aligned}
$$

Equation (4) models the general case. Next, we will cast (4) into a simpler formulation suited to our particular application.

\section{B. Case study}

Let us assume a cubic nonlinearity with cubic stiffness $\gamma$, small static displacement $\left(x_{0}<<A\right)$ and a linear force $F^{\theta}(x)=f$. Under these assumptions, $\theta=(f, \gamma), G^{\theta}(x)=-\gamma \omega_{0}^{2} x^{3}$ and:

$$
\begin{aligned}
& h\left(A, \omega, \omega_{0}, Q, f, \gamma\right)= \\
& 1-\frac{A^{2}}{f^{2}}\left[\left(\omega_{0}^{2}-\omega^{2}+\frac{3}{4} \gamma \omega_{0}^{2} A^{2}\right)^{2}+\frac{\omega^{2} \omega_{0}^{2}}{Q^{2}}\right]
\end{aligned}
$$
(5).

In the following sections, we present results obtained with

\section{Mathematical formulation of characterization problems}

Let us consider a set of $N$ measurements $\left(A_{k}, \omega_{k}\right)_{k \in[1 ; \mathrm{N}]}$ obtained by sweeping $\omega$ at a constant actuation amplitude. Our aim is to identify, with this data, the values of $\omega_{0}, Q$ and $\theta$ to characterize the resonator. A general characterization method consists in minimizing:

$$
H\left(\omega_{0}, Q, \theta\right)=\sum_{k=1}^{N}\left[h\left(A_{k}, \omega_{k}, \omega_{0}, Q, \theta\right)\right]^{2}
$$

where $h$ is defined by (4). This criterion yields quick and accurate convergence, as illustrated in Section III.

\section{SIMULATION RESULTS}

Given the previous mathematical analysis, we develop a characterization procedure. We show how to fit a 4-parameter nonlinear frequency response via the minimization of (6) and illustrate our method on a resonator described by (5).

\section{A. Assumptions}

We present a few sets of relevant simulations which highlight that the minimization of $H$ leads to an accurate identification procedure. Several values of $Q, f$ and $\gamma$ defined in (5) are considered (see Table I) with a natural frequency $\omega_{0}$ normalized to unity.
TABLE I. SIMULATION PARAMETER VALUES

\begin{tabular}{|c|c|c|c|c|c|c|}
\hline Parameter & \multicolumn{5}{|c|}{ Values } \\
\hline$\gamma$ & $-3 \times 10^{-3}$ & $-10^{-3}$ & $-5 \times 10^{-4}$ & $5 \times 10^{-4}$ & $10^{-3}$ & $3 \times 10^{-3}$ \\
\hline$Q$ & $10^{3}$ & $4 \times 10^{3}$ & $8 \times 10^{3}$ & $10^{4}$ & $2 \times 10^{4}$ & \multicolumn{1}{|c}{} \\
\cline { 1 - 5 }$f$ & $10^{-4}$ & $10^{-3}$ & $3.3 \times 10^{-3}$ & $5 \times 10^{-3}$ & \multicolumn{1}{|c}{} \\
\cline { 1 - 5 } & &
\end{tabular}

Then, we solve (5) for all the combinations of $\left(\omega_{0}, Q, f, \gamma\right)$. For each combination, we generate a set of $N=30$ pseudoexperimental measurements $\left(A_{k}, \omega_{k}\right)_{k \in[1 ; \mathrm{N}]}$ of a frequency sweep response close to resonance. For the identification procedure, we assume that the experimenter has enough a priori knowledge to guess $\omega_{0}$ in the range $\left[\omega_{0}(1-10 / Q), \omega_{0}(1+10 / Q)\right]$. For values of $\gamma$ larger than the ones considered in Table I, this interval must be extended so that the jump discontinuity of the amplitude response remains within the sweep range. Nevertheless, enlarging this interval to include the jump discontinuity has no effect in the fitting procedure. Finally, we assume that the experimenter has also a priori information on the values of $Q, f$ and $\gamma$ within a range of $\pm 100 \%$.

\section{B. Proposed approach}

For each set of $\left(\omega_{0}, Q_{,}, \gamma\right)$, we run 10000 nonlinear leastsquares fits, using a trust-region algorithm with a lower bound $[0,0,0,0]$. Each of the 10000 tests corresponds to a different initial condition $\left(\omega_{0 \mathrm{i}}, Q_{i} f_{i}, \gamma_{i}\right)$. In order to limit the computation time, we set the maximum number of iterations at 100 and the maximum number of function evaluations at 1000 . For every parameter set $\left(\omega_{0}, Q, f, \gamma\right)$, around $92 \%$ of the 10000 tests converge to a local minimum, the remaining $8 \%$ stopping prematurely due to an excessive number of iterations or function evaluations. In these cases, $H$ keeps decreasing too slowly and more than 10000 iterations and function evaluations would be needed to approach a minimum.

To avoid this outcome, we developed a recursive algorithm, given in Fig. 1, which converges within 2 cycles (i.e. max 200 iterations and 2000 function evaluations) in $1-0.08^{2}>99 \%$ of cases.

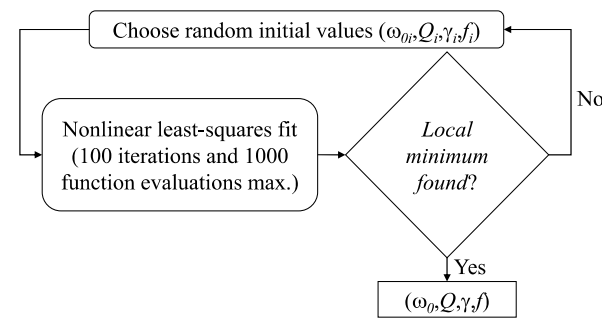

Fig. 1. Flow-chart of the recursive procedure. After 2 cycles, the success rate is already greater than $99 \%$.

\section{Accuracy of the solution}

In order to validate the optimization procedure, we check if the local minimum found is accurate, i.e. if the parameters are accurately identified. In all the simulations with the initial conditions and parameter values considered in Sub-Section 
III.A., the algorithm converges to the expected values, with a very small residual function $H\left(<10^{-13 \%}\right)$. To illustrate this precision, Fig. 2 shows the simulated results (obtained by solving $h\left(A, \omega, \omega_{0}, Q, f, \gamma\right)=0$ for each given set of $\left.\left(\omega_{0}, Q_{2}, \gamma\right)\right)$ and the fit obtained after the nonlinear least-squares curve fitting procedure described in Fig. 1. For the sake of readability, we only report in Fig. 2 the results obtained with $\omega_{0}=1, Q=10000$, $f=3.3 \times 10^{-3}, \gamma=-3 \times 10^{-3}, \gamma=-1 \times 10^{-3}, \gamma=-5 \times 10^{-4}$ and $\gamma=3 \times 10^{-3}$. All the simulations lead to a relative error lower than $10^{-5 \%}$ on each estimated parameter. These results show that the parameters are accurately extracted.

\section{APPLICATION TO CAPACITIVE MEMS RESONATORS}

In this section, we show how to characterize a capacitive clamped-clamped MEMS resonator in nonlinear regime and demonstrate our method on an experimental setup.

\section{A. Theoretical background}

The force applied on the resonator of a clamped-clamped resonator subject to the electrostatic force may be approximated by [10]:

$$
F \approx \frac{\varepsilon S I_{0}\left(V_{b}^{2}+2 V_{b} V_{a c t} \sin (\omega t+\varphi)\right)}{2 G^{2}(1-x)^{3 / 2}} \text { with } I_{0}=\int_{0}^{1} w_{c c}=\frac{1}{2}
$$

Where $w_{c c}(x)=\sin (\pi x)^{2}$ is the approximated shape of the clamped-clamped mode, $V_{b}$ the bias voltage, $V_{a c t}$ the actuation voltage $\left(V_{b}>>V_{a c t}\right), S$ the surface of the plane electrodes at rest, $G$ the gap between the two electrodes and $\varepsilon$ the permittivity of the material between the two electrodes.

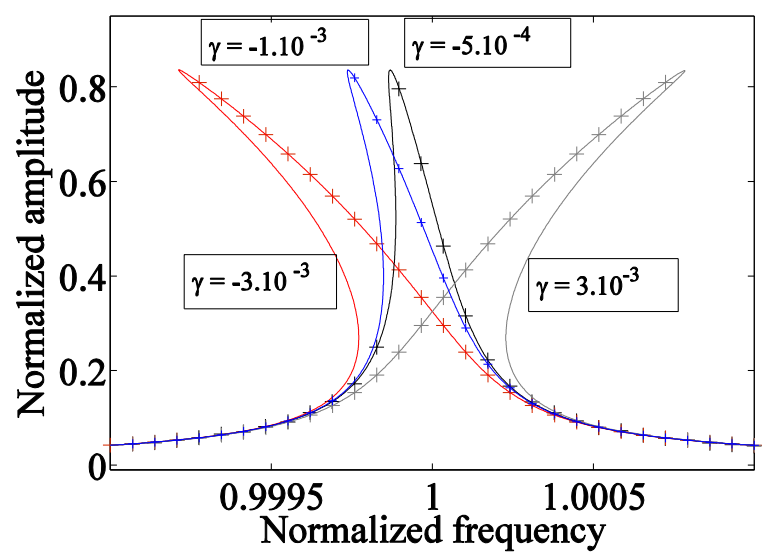

Fig. 2. Typical curves: simulated frequency responses (crosses) and corresponding nonlinear least-squares fits $\left(\omega_{0}=1, Q=10000, f=3.3 .10^{-3}\right)$.

Assuming that the total displacement of the resonator is largely inferior to the gap $(x<<1)$, a series expansion about $x=0$ of the dynamic response of a second-order resonator yields:

$$
\frac{d^{2} x}{d t^{2}}+\frac{\Omega_{0}}{Q_{e}} \frac{d x}{d t}+\Omega_{0}^{2}\left(x+\frac{6 \gamma}{7} x^{2}+\gamma x^{3}\right)=f_{s}+f_{1} \sin \omega t
$$

with $\gamma=-\frac{35 \varepsilon S V_{b}^{2} I_{0}}{32 \Omega_{0}^{2} m G^{3} I_{1}}, \Omega_{0}^{2}=\frac{35 \omega_{0}^{2}}{35-24 \gamma}, f_{s}=-\frac{8 \gamma \Omega_{0}^{2}}{35}$, $f_{1}=-\frac{32 \gamma \Omega_{0}^{2} V_{a c t}}{35 V_{b}} \quad, \quad Q_{e}=Q \sqrt{1+\frac{24 \gamma \Omega_{0}^{2}}{35 \omega_{0}^{2}}}$

$I_{1}=\int_{0}^{1} w_{c c}^{2} \approx 0.397$ and $m=m_{t o t} \int_{0}^{1} w_{c c}^{2}$ for a beam of total mass $m_{t o t}\left(m=3 m_{t o t} / 8\right)$.

Let us write $x(t)=A \sin \omega t$. The method of harmonic balance as in Sub-Section II.A. yields:

$$
\begin{aligned}
& h\left(A, \Omega, \Omega_{0}, Q_{e}, \gamma\right)= \\
& 1-\frac{35^{2} A^{2} V_{b}^{2}}{32^{2} V_{a c t}^{2} \gamma^{2} \Omega_{0}^{4}}\left[\left(\Omega_{0}^{2}-\Omega^{2}-\frac{3}{4} \gamma \Omega_{0}^{2} A^{2}\right)^{2}+\frac{\Omega^{2} \Omega_{0}^{2}}{Q_{e}^{2}}\right]=0
\end{aligned}
$$

Equation (8) is only valid as long as the displacement of the resonator is largely inferior to the gap distance. Outside the scope of this assumption, more tedious calculations yield the expression of $h$. Some of them are reported in [11].

\section{B. Parameter extraction as a minimization problem}

Let us now consider a set of $N$ experimental measurements $\left(V_{k}, \Omega_{k}\right)$ for $k=1$ to $N$ and write:

$$
H_{e}\left(G_{e}, \Omega_{0}, Q_{e}, \gamma\right)=\sum_{k=1}^{N}\left[h_{e}\left(\frac{V_{k}}{G_{e}}, \Omega_{k}, \Omega_{0}, Q_{e}, \gamma\right)\right]^{2}
$$

with an electric gain $G_{e}$. To characterize the resonator, we minimize $H_{e}$ with respect to $G_{e}, \Omega_{0}, Q_{e}$ and $\gamma$. Compared to Section III, the unknowns are now $G_{e}, \Omega_{0}, Q_{e}$ and $\gamma$ due to the relationship between $f_{l}$ and $\gamma$.

In Section $\mathrm{V}$, we describe our experimental setup and show how to characterize a MEMS resonator described by (8).

\section{EXPERIMENTAL RESULTS}

\section{A. Experimental setup}

The resonator experimentally characterized in this study was originally developed by SEXTANT Avionics (currently THALES) [12]. It is industrially assembled by the fusionbonding of three etched silicon wafers and consists of a resonant clamped-clamped beam resting on a rectangular diaphragm. During the manufacturing process, the beam is encapsulated in vacuum to achieve a high mechanical Q-factor $\left(Q \approx 1.9 \times 10^{4}\right.$ at $V_{b}=15 \mathrm{~V}$ and $\left.V_{a c t}=10 \mathrm{mV}\right)$. The natural frequency $f_{0}=\omega_{0} / 2 \pi$ of the device is close to $68 \mathrm{kHz}$.

A bias voltage $V_{b}=60 \mathrm{~V}$ and an actuation voltage $V_{a c t}$ are applied to the resonator in a setup described in [13] where $V_{a c t}=10 \mathrm{mV}$ and $25 \mathrm{mV}$. With this setup, the frequency response of the resonator can be obtained without parasitic current effects [13].

\section{B. Experimental frequency response}

Fig. 3 reports the two experimental low-noise sets of measurements and the fitted curves after the nonlinear leastsquares fitting procedure explained in Section III, where the normalized frequency is defined as $\Omega / \Omega_{0}$. The fitted curves 
highlights a very good agreement between the model and experimental results. Compared to [6,7], Fig. 3 confirms that the fitting procedure requires very few experimental measurements around the maximal amplitude. The actuation frequency is only swept down, corresponding to the upper branch of the response and, hence, to a better SNR than the lower branch.

The fitting procedure yields the values of $G_{e}, F_{0}=\Omega_{0} / 2 \pi, Q_{e}$ and $\gamma$. We deduce $Q=1.22 \times 10^{4}$ at $V_{a c t}=10 \mathrm{mV}$ and $Q=1.02 \times 10^{4}$ at $V_{a c t}=25 \mathrm{mV}, G_{e}=1.32 \mathrm{~V}, f_{0}=\omega_{0} / 2 \pi=68.25 \mathrm{kHz}$. This resonant frequency agrees well with the expected value [13]. One can notice that the Q-factor decreases largely as the actuation voltage (hence amplitude) grows. Since this resonator exhibits parametric resonance at high bias voltages [14], we believe that this decrease is related to this phenomenon. In Fig.3, $\max (x)=0.14$ (at $V_{a c t}=25 \mathrm{mV}$ ), sufficiently small for the thirdorder Taylor series expansion of Subsection IV.A.

The values of $G_{e}$ and $\gamma$ give valuable information about the structure of the resonator which can hardly be measured via other methods since our sensor is encapsulated in an opaque vacuum package.
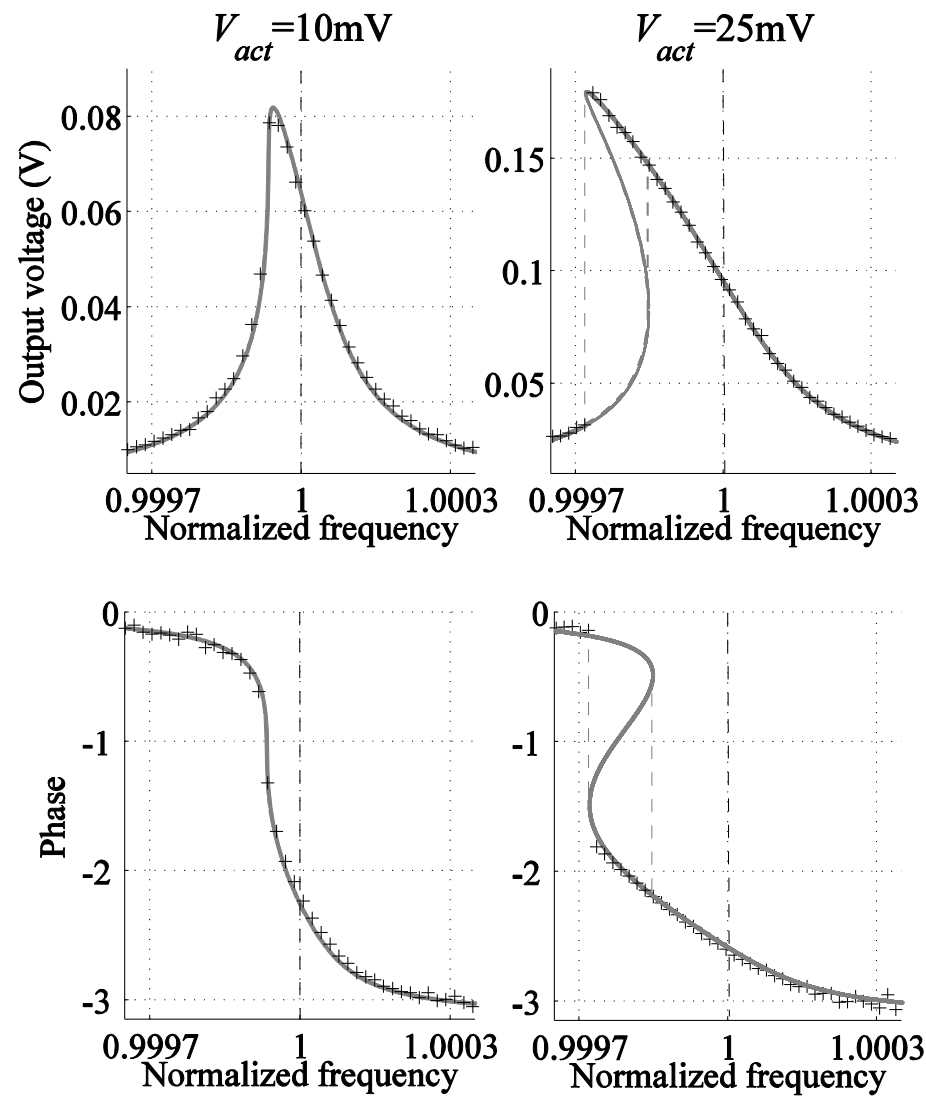

Fig. 3. Experimental nonlinear frequency responses (dark crosses) and fitted curves (grey lines) after nonlinear least-squares fitting for $V_{a c t}=10 \mathrm{mV}$ (left) and $V_{\text {act }}=25 \mathrm{mV}$ (right).

\section{CONCLUSION}

The results presented in this paper show that nonlinear open-loop frequency responses allow parameter extraction without demanding measurements around the maximal amplitude and around the eventual bifurcations and/or jump discontinuities. We gave the theoretical background for our technique and the algorithm steps used to carry out the parameter extraction. We applied our method to a capacitive MEMS resonator and obtained experimental results showing its accuracy. The information extracted from nonlinear frequency responses may be used to evaluate the condition of a resonator, paves the way to a better understanding of resonant systems and offers promising developments for industrial and maintenance applications. Future work will aim at experimental validation of our technique to resonators subject to both hardening and softening nonlinearities as well as lifetime management of sensors used in harsh environments.

\section{REFERENCES}

[1] S. S. Rao, Mechanical Vibrations, 3rd ed. Reading, MA: AddisonWesley, 1995, p. 204.

[2] Z. Zeng, M.A.P. Pertijs and D. M. Karabacak, An energy-efficient readout circuit for resonant sensors based on ring-down measurements, Review of Scientific Instruments, vol. 84, 2013.

[3] R. Modlinski, A. Witvrouw et al., Creep as a reliability problem in MEMS, Microelectronics Reliability, vol. 44, pp. 1733-1738., 2004.

[4] T. Ando, M. Shikida and K. Sato, Tensile-mode fatigue testing of silicon films as structural materials for MEMS, Sensors and Actuators A, vol. 93, pp. $70-75,2001$.

[5] J. Wibbeler, G. Pfeifer and M. Hietschold, Parasitic charging of dielectric surfaces in capacitive microelectromechanical systems (MEMS), Sensors and Actuators A, vol. 71, pp. 74-80, 1998.

[6] W. O. Davis, Measuring Quality Factor From a Nonlinear Frequency Response With Jump Discontinuities, J. of Microelectromechanical systems, vol. 20, pp. 968-975, 2011.

[7] A. J. Dick, B. Balachandran, D. L. DeVoe and C. D. Mote Jr, Parametric identification of piezoelectric microscale resonators, J. Micromech. Microeng., vol. 16, pp. 1593 - 1601, 2006.

[8] R. Lifshitz and M. C. Cross, Nonlinear Dynamics of Nanomechanical and Micromechanical Resonators, Review of Nonlinear Dynamics and Complexity,. NJ: Wiley, 2008, pp. 2-17

[9] A. Gelb and W. E. Vander Velde, Multiple-input describing functions and nonlinear system design, NY: McGraw-Hill, 1968, pp. 47-55.

[10] J. Juillard, G. Arndt and E. Colinet, Modeling of Micromachined Beams Subject to Nonlinear Restoring or Damping Forces, J. of Microelectromechanical systems, vol. 20, pp. 165-177, 2011.

[11] A. Brenes, J. Juillard, L. Bourgois, F. Vinci Dos Santos, Characterization of capacitive MEMS resonators via nonlinear openloop frequency responses, Proceedings of DTIP, 2015.

[12] J. Mandle, O. Lefort and A. Migeon, A new micromachined silicon highaccuracy pressure sensor, Sensors and Actuators A, vol. 46-47, pp. 129-132, 1995

[13] A. Brenes, J. Juillard, F. Vinci Dos Santos and A. Bonnoit, Characterization of MEMS resonators via feedthrough de-embedding of harmonic and subharmonic pulsed-mode response, Sensors and Actuators A, "in press".

[14] A. Brenes, J. Juillard and F. Vinci Dos Santos., Electrostaticallyinduced modal crosstalk phenomena in resonant MEMS sensors, Proceedings of DTIP, pp. 294-297, 2014. 
